\title{
Transforming YouTube into a valid source of knowledge for Anatomy students
}

\author{
Antoni Alegre-Martínez1, María Isabel Martínez-Martínez², José Luis Alfonso- \\ Sanchez ${ }^{3}$ \\ ${ }^{1}$ Biomedical Sciences department, Faculty of Health Sciencies, Cardenal Herrera CEU \\ University, Spain ${ }^{2}$ Public Health department, Faculty of Nursing and Podiatry, University \\ of Valencia, Spain. ${ }^{3}$ Department of Preventive Medicine. University of Valencia, Spain.
}

\begin{abstract}
YouTube is a free and easily accessible tool, with growing importance in the teaching field due to the content of the videos and their interaction options through comments, responses and insertion in social networks. However, some limitations can reduce the value of this tool in University teaching if institutional control is not carried out. Our project consists of the search for experiences based on learning Anatomy on YouTube to be able to incorporate this tool in our department. Almost all researchers found that most of students use YouTube as a source of anatomical knowledge, despite limitations and criticism based on ethical and privacy issues, the video experience itself, the YouTube search algorithm, lack of quality control, advertising purposes or excessive video offer. Researchers experienced that most of the available videos had a poor quality and many mistakes, so professors must be involved in the search and selection of the best appropriate videos. We conclude that YouTube can be used as a source of knowledge for anatomical learning. However it is necessary to inform students of the inconveniences and risks, and make a critical selection by the professors of the videos that best fit in the teaching program.
\end{abstract}

Keywords: YouTube learning Anatomy videos reliability dissection. 


\section{Introduction}

The teaching of medicine has followed a practically constant pattern during centuries until the emergence of Internet that have provided doctors, educators and students with smartphone applications, social networks, blogs and media exchange (Farnan et al., 2008). Multimedia resources had a special impact, especially video, which allows students to control the extent of assimilated knowledge and the way of presenting it, with a series of tools that allow them to pause, rewind and re-view the topics according to their own rhythm of content acquisition (Patel et al., 2015). Conventional Anatomy teaching methods have evolved into combined learning that incorporates multimedia and other technological advances that support different formats of combined learning (Barry et al., 2016). The aim of our reseach focuses on dissection videos for teaching Anatomy in medical schools, because the face-to-face dissection courses do not represent a uniform learning experience, with different students directing the practice in a different way of dissection, sometimes in divergent ways (Bergman, 2015). There are virtual models that allow students to navigate instantly and remove layers of tissue to observe for example the path of a nerve, however they require expensive licenses that not all universities are willing to assume (Bergman, 2015).

These new technologies pose challenges for professors and educators, but also opportunities (Farnan et al., 2008). The creation of models and videos represent an additional time and effort for teachers that is not always possible to face by the institution (Jaffar, 2012). Teachers should recognize the importance of video sharing sites like YouTube in education and invest in the use of Web 2.0. to apply their possibilities in teaching (Azer, 2012). Unlike the tools described above, YouTube is a free of charge and worldwide available webpage, for which access is enough with a computer, tablet or smartphone with an Internet connection (Bergman, 2015).

Our Anatomy Department asks whether it is useful or not to recommend certain YouTube videos to students. The aim of our research focuses on perform searches with an institutional filter to incorporate this tool in the learning of the structure of the human body.

\section{Objectives}

Our goal is to conduct a bibliographic search for research on student/teacher experiences in the last decade of using YouTube to learn/teach Anatomy,to be able to incorporate this tool to the teaching of Anatomy in our department. 


\section{Material and Methods}

We conducted a bibliographic search in Pubmed and Google Scholar including the keywords "youtube", "Anatomy", "videos", "learning" to find experiences of incorporating the video portal to anatomical teaching. The detailed reading of the results allowed us to establish conclusions to consider incorporating YouTube videos in the teaching of Anatomy in our department.

\section{Results}

\subsection{YouTube use by Anatomy students}

YouTube is able to offer updated educational resources aimed at medical students by adding not only the multimedia content itself, but the additional functionality of providing interactive media among users: students can add comments, answer to them and exchange videos with each other (Rabee et al., 2015). Students of the new generations actively interact with social networks to integrate them into their educational experience, participating with the course materials and sharing their knowledge, often waiting for immediate responses and comments (Barry et al., 2016). There have been several studies that have explored the percentage of Health Sciences students who use YouTube as an educational resource:

- Rapp et al. (2016) conducted a study in the state of Iowa where 90\% of respondents reported watching videos for their surgery classes, being YouTube the most used source among respondents $(86 \%)$.

- Hulme et al. (2017) cite that $78 \%$ of the students who used web platforms to obtain information used YouTube as their main source of video.

- Patel et al. (2015) asked to 91 second-year medical students if they used Internetbased resources as a teaching source for the Anatomy subject, stating that $98 \%$ used YouTube as a source of information, and $92 \%$ found Anatomy videos beneficial for their learning.

- Rodriguez et al. (2018) reported that $95 \%$ of surgery residents used YouTube to face surgery and investigate cases, especially when learning a specific technique; compared to $25 \%$ who used the SCORE website. However, they confessed that most of them did not investigate beyond the first options shown on the first page of the search engine.

- Jaffar (2012) found that $98 \%$ of their students used YouTube as a source to gain knowledge of Anatomy applied to medicine.

- Barry et al. (2016) asked their students about the method they used to learn Anatomy, finding that $34 \%$ of students watched anatomical videos once a month, $26 \%$ once a week, $16 \%$ once a semester, $14 \%$ always and $10 \%$ three times per 
week. The utility found by the students was very high, with $78 \%$ qualifying them as useful, although $22 \%$ indicated that the utility varied depending on the content.

\subsection{Challenges and disadvantages of YouTube as a learning tool}

The main criticisms found in the literature can be grouped into:

- General criticisms of video Anatomy learning:

- Self-study is not always beneficial for the student, since it is necessary to have the guidance and guardianship of experts in Medicine and Anatomy (Bergman, 2015).

- The video is not able to report on the texture of the tissues and the kinesthetic aspects that corpse dissection does (Patel et al., 2015).

- The Anatomy videos available on the Internet do not always raise awareness about the ethical problems inherent in the filming of corpses, such as the origin of the videos, the subject's permission for filming and distribution online. It is necessary the discussion in classroom of this ethical issues (Barry et al., 2016).

- On many occasions, the students themselves record these videos to upload to their social networks. Even if the intention is educational, it is questionable whether these students have had the permission of the institutional authority for these recordings. Therefore, students should be cautious to avoid these non-professional behaviors (Raikos \& Waidyasekara, 2014).

- Criticisms regarding the use of social networks for learning:

- Many students and teachers do not feel comfortable using social media platforms, which can disturb the teacher-student relationship. In many cases, privacy issues also arise with the accounts associated with these platforms (Barry et al., 2016).

- Criticisms regarding how YouTube works as a platform:

- YouTube videos are shown by popularity when applying a patented algorithm that includes the count of visits, user history and comments, without taking into account the quality, usefulness or veracity (Rodriguez et al. , 2018).

- Videos are usually uploaded without quality control, finding unregulated, inaccurate, misleading or biased content (Raikos \& Waidyasekara, 2014).

- The indiscriminate upload of videos by any user prevents new generations from knowing the ethical problems related to the use of web-based videos whose origin is unknown (Barry et al., 2016).

- In many cases, YouTube videos aim to promote and publicize teaching institutions or hospitals, rather than teaching students (Lee et al., 2015).

- Overcrowded feeling, with too many videos, many of them of low quality, confusing students without knowing where to look (Rabee et al., 2015).

- Some students may find YouTube as a challenging, slow and frustrating platform (Bergman, 2015). 


\subsection{Reliability of YouTube anatomical videos}

Is it reliable to adress students to YouTube for use it in their knowledge acquisition? Without a guide to specific links to certain videos, it is risky due to the uncontrolled and potentially erroneous content available (Hulme \& Strkalj, 2017). The publication of a list of reference links could overcome the credibility challenges of YouTube, which is one of its main drawbacks, as well as saving a lot of time for students and encouraging them to go to this platform: it was reported a $19 \%$ increase in the use of YouTube after the publication of the list of links provided by the professors in a study performed by Jaffar (Jaffar, 2012).

In addition, today's students should be taught where to find information and how to discriminate between thousands of sources what is appropriate for learning, so it is needed to provide students with skills to critically evaluate resources such YouTube (Rodriguez et al., 2018). It is important that professors carefully examine the suggested links to determine their adequacy to the content of the subject and their absence of errors (Jaffar, 2012). Several studies have analyzed the videos about Anatomy available on YouTube (some of them had a surgical orientation) to perform a critical analysis on them:

- Lee et al. (2015) evaluated 100 YouTube videos covering 25 hours of content on ten specific topics, observing the first ten videos to appear on each topic. Only 1 video of the 100 analyzed could be classified as "good". 60\% of videos about venous puncture or cardiopulmonary resuscitation were classified as "satisfactory", showing a need for a rigorous evaluation of the quality of medical videos on YouTube. Lee also analyzed 73 videos of laparoscopic cholecystectomy, of which $11(15.1 \%)$ were evaluated as good, $40(54.8 \%)$ moderate and $22(30.1 \%)$ poor. Despite these results, they highlighted the great potential educational value that these videos can have on students, doctors in training, patients and their families.

- Fischer et al. (2013) analyzed videos on knee arthrocentesis. 13 videos met the inclusion criteria, but only 8 were considered useful for didactic purposes $(62 \%)$. Surprisingly, 6 videos $(46 \%)$ had a deficiency in the sterility of the technique. The team concludes that it is necessary to improve the teaching materials based on YouTube before recommending them to students

- Azer (2012) performed the search by minutes, analyzing 216 minutes of surface Anatomy content available on YouTube, finding that only 59 minutes (27\%) were useful for their students' learning. As optimistic data, the most useful videos had an average views that doubled that of the unhelpful videos. Azer also analyzed 356 comments to check if they could be useful to other students, finding that only 151 were useful, raising some clues for the creators to improve their presentations, for example in technical sections (background noise, camera type), etc. Most of these comments stood out for their brevity. 
- Bergman (2015) found that only $25 \%$ of the videos analyzed included an adequate and valid anatomical content, considering that the professor must be involved in the search and selection of the best appropriate videos related to the subject.

- Derakhshan et al. (2019) studied videos on facial surgery concluding that they are deficient with respect to the criteria for surgical indication, patient selection or possible complications, with notable absence of intraoperative reference points (for example, the facial nerve or the type of incision was not adequately discussed) or discussion of the key principles. They concluded that until the quality of these videos was improved, they could not recommend them as a complement to their students' learning.

- Rodríguez et al. (2018) analyzed the videos of laparoscopic cholecystectomy available on YouTube and were optimistic to find that the techniques were adequate and with reasonable levels of technical competence.

- Raikos \& Waidyasekara (2014) observed that the videos of the Anatomy of the heart that were on YouTube poorly transmitted the anatomical knowledge, Only $25.9 \%$ of the videos were able to approve according to their scoring system. The authors attribute it to the fact that many of these videos are made by the students themselves with lack of planning, without a narrative script and with insufficient anatomical knowledge. Therefore it is recommended to underestimate the educational use of YouTube to improve student learning.

\subsection{Student experience with the guided use of YouTube videos for anatomical learning}

Some institutional YouTube channels can improve the teaching experiences. Jaffar stated that $92 \%$ of the students who used the "Human Anatomy Education" channel agreed that this method helped them in anatomical learning, increase understanding of structures, remembering 3D images, and valued availability 24 hours a day (Jaffar , 2012). Numerous studies advance in this line:

- Mukhopadhyay et al. (2014) uploaded 40 videos on dental Anatomy and local anesthesia available to students for more than a year, with a total of 71,000 reproductions especially from the United States and Australia, with an increasing number of visits originating in developing countries.

- Azer et al. (2013) They found 20 relevant videos for cardiovascular system exams and 36 for respiratory system exams. After further screening, the number fell respectively to 9 and 7 . Although it is a small number, they can be used by medical students in their autonomous learning.

- Students also recommended video lists including their guidelines for when to use them, such embedding them in blogs or personal pages. Students valued that the videos were among the first to appear in the searches and assessed that the list improved semester after semester (Berk, 2009). 
- Welbourne \& Grant (2016) highlighted the difficulty of capturing the attention of the public to reach as many students as possible, so it was necessary to understand the factors contributing to the growth of the popularity of channels and videos to ensure that the message is distributed to consolidate long-term success . It is important that communicators present their real face and communicate with the community, avoiding the error of thinking that YouTube is a mere video store since interaction with students and their participation is their true essence.

- Michael L. Wesch, a professor at Kansas State University, wanted to demonstrate the scope of a video about a conference, publishing a video that reached 400,000 views, compared to the few dozen people who attended his lesson in a master class, or a few thousand readers in the case to have been published in a journal. YouTube must be seen as a new way for teachers to communicate and reach a larger audience. "It's easier than people think," Wesch said about making videos online (Young, 2008).

\section{Conclusions}

After the contrast of the experiences, we conclude that YouTube can be used as a source of knowledge for anatomical learning, but for this it is necessary to inform students of the inconveniences and risks, and make a critical selection by the professors of the videos that best fit in the teaching program.

\section{References}

Azer, S. A. (2012). Can "YouTube" help students in learning surface Anatomy? Surgical and Radiologic Anatomy, 34(5), 465-468.

Azer, S. A., AlGrain, H. A., AlKhelaif, R. A., \& AlEshaiwi, S. M. (2013). Evaluation of the educational value of YouTube videos about physical examination of the cardiovascular and respiratory systems. Journal of Medical Internet Research, 15(11), e241.

Barry, D. S., Marzouk, F., Chulak- Oglu, K., Bennett, D., Tierney, P., \& O'Keeffe, G. W. (2016). Anatomy education for the YouTube generation. Anatomical Sciences Education, 9(1), 90-96.

Bergman, E. M. (2015). Discussing dissection in Anatomy education. Perspectives on Medical Education, 4(5), 211-213.

Berk, R. A. (2009). Multimedia teaching with video clips: TV, movies, YouTube, and $\mathrm{mtv} \mathrm{U}$ in the college classroom. International Journal of Technology in Teaching \& Learning, 5(1)

Derakhshan, A., Lee, L., Bhama, P., Barbarite, E., \& Shaye, D. (2019). Assessing the educational quality of 'YouTube'videos for facelifts. American Journal of Otolaryngology, 40(2), 156-159. 
Duncan, I., Yarwood-Ross, L., \& Haigh, C. (2013). YouTube as a source of clinical skills education. Nurse Education Today, 33(12), 1576-1580.

Farnan, J. M., Paro, J. A., Higa, J., Edelson, J., \& Arora, V. M. (2008). The YouTube generation: Implications for medical professionalism. Perspectives in Biology and Medicine, 51(4), 517-524.

Fischer, J., Geurts, J., Valderrabano, V., \& Hugle, T. (2013). Educational quality of YouTube videos on knee arthrocentesis. Journal of Clinical Rheumatology : Practical Reports on Rheumatic \& Musculoskeletal Diseases, 19(7), 373-376. doi:10.1097/RHU.0b013e3182a69fb2 [doi]

Hulme, A., \& Strkalj, G. (2017). Videos in Anatomy education: History, present usage and future prospects. International Journal of Morphology, 35(4)

Jaffar, A. A. (2012). YouTube: An emerging tool in Anatomy education. Anatomical Sciences Education, 5(3), 158-164.

Lee, J. S., Seo, H. S., \& Hong, T. H. (2015). YouTube as a potential training method for laparoscopic cholecystectomy. Annals of Surgical Treatment and Research, 89(2), 9297.

Mukhopadhyay, S., Kruger, E., \& Tennant, M. (2014). YouTube: A new way of supplementing traditional methods in dental education. Journal of Dental Education, 78(11), 1568-1571. doi:78/11/1568 [pii]

Patel, S. B., Mauro, D., Fenn, J., Sharkey, D. R., \& Jones, C. (2015). Is dissection the only way to learn Anatomy? thoughts from students at a non-dissecting based medical school. Perspectives on Medical Education, 4(5), 259-260.

Rabee, R., Najim, M., Sherwani, Y., Ahmed, M., Ashraf, M., Al-Jibury, O., Ahmed, A. (2015). YouTube in medical education: A student's perspective. Medical Education Online, 20, 29507. doi:10.3402/meo.v20.29507 [doi]

Raikos, A., \& Waidyasekara, P. (2014). How useful is YouTube in learning heart Anatomy? Anatomical Sciences Education, 7(1), 12-18.

Rapp, A. K., Healy, M. G., Charlton, M. E., Keith, J. N., Rosenbaum, M. E., \& Kapadia, M. R. (2016). YouTube is the most frequently used educational video source for surgical preparation. Journal of Surgical Education, 73(6), 1072-1076.

Rodriguez, H. A., Young, M. T., Jackson, H. T., Oelschlager, B. K., \& Wright, A. S. (2018). Viewer discretion advised: Is YouTube a friend or foe in surgical education? Surgical Endoscopy, 32(4), 1724-1728.

Welbourne, D. J., \& Grant, W. J. (2016). Science communication on YouTube: Factors that affect channel and video popularity. Public Understanding of Science, 25(6), 706-718.

Young, J. R. (2008). YouTube professors-scholars as online video stars. Education Digest, 73(9), 14. 